\title{
Users' emotional evaluation towards kansei-based higher learning institution website using geneva emotion wheel
}

\author{
Punitha Turumugon ${ }^{1}$, Aslina Baharum ${ }^{2}$, Nadia Hanin Nazlan ${ }^{3}$, Nor Azida Mohamed Noh', \\ Noorsidi Aizuddin Mat Noor ${ }^{5}$, Emelia Abdul Rahim ${ }^{6}$ \\ ${ }^{1,2}$ Faculty of Computing and Informatics, Universiti Malaysia Sabah, Malaysia \\ ${ }^{3}$ Faculty of Hotel \& Tourism Management, Universiti Teknologi MARA Cawangan Selangor, Malaysia \\ ${ }^{4}$ Faculty Computer and Mathematical Sciences, UiTM Cawangan Melaka, Malaysia \\ ${ }^{5}$ UTM CRES, Faculty of Built Environment and Surveying, Universiti Teknologi Malaysia, Malaysia \\ ${ }^{6}$ Faculty of Business Management, Universiti Teknologi MARA Cawangan Sarawak, Malaysia
}

\begin{tabular}{l} 
Article Info \\
\hline Article history: \\
Received Mar 1, 2019 \\
Revised Jun 17, 2019 \\
Accepted Jul 11, 2019 \\
\hline
\end{tabular}

\section{Keywords:}

Kansei engineering

Standard

User interface design

\begin{abstract}
Functionality, usability, and pleasure are the three elements that influence consumers decision-making and behavioral intentions. This study attempts to fill in the gap in the web design literature by justifying the Kansei-based engineering technique by embedding emotional cues in Higher Learning website designs. This study aims to test the effectiveness of a Kansei-based website that was designed in accordance with the Kansei-based standards for web design. Using an online Geneva Emotion Wheel survey, the results suggest that the Kansei-based website elicit favorable positive emotion from users.
\end{abstract}

\section{Corresponding Author:}

Aslina Baharum,

Faculty of Computing and Informatics,

Universiti Malaysia Sabah, 88400 Kota Kinabalu, Sabah, Malaysia.

Email: aslina@ums.edu.my

\section{INTRODUCTION}

Designs with emotional elements aims to produce products that evoke users' positive emotion $[1,2]$. Research suggested that emotion affects users' perception, decision making [3, 4] and simultaneously entices users to engage with the website $[5,6]$. Websites with emotionally engaging elements has the potential to facilitate website revisit intention and loyalty [7]. Users can be emotionally engaged with a website that has affective user interface that stimulates their experience and satisfies their emotional needs. In contrast, websites that are poorly designed tend to arouse negative emotions such as anxiety, confusion, and frustration. Users become susceptible to negative emotions when they encounter difficulties in navigating the website due to poorly designed interface [7]. Therefore, it is crucial to embed emotional elements that can encourage joy, pleasure, and interest. To elicit positive emotional response from the users, web designers need to identify users' affective needs. Studies have reported that users' emotional needs can be linked and translated into design elements that are able to trigger emotional engagement $[8,9]$. However, the major challenge lies in understanding users' emotional needs and transforming these needs into design features that create an 'emotional bridge' between the product and the user [10]. Moreover, past study [11] have reported that web interface designers are facing a challenge in establishing a standard for web interface designs embedded with emotion-evoking cues [11].

Due to the influential nature of the emotion-evoking web designs, a recent study [1] employed Kansei engineering technique on user interface design for higher learning institution website in Malaysia. 
The study was inspired by previous research on Kansei engineering technique on web design for e-learning [12], e-commerce [13], job hunting sites [11], student blogs [6], university departments website [3] and university websites [9]. The study [1] attempted to elicit users' emotions by incorporating emotion-inducing elements in a higher learning institution website. A standard for Kansei-based web design for Higher Learning institution was also proposed in study [1].

In an attempt to extend previous literature [1], the current study seeks to justify the Kansei-based web design standards through the development of a Kansei-based prototype that enables the evaluation of users' emotional experience using a self-reported assessment tool, the Geneva Emotion Wheel (GEW). The evaluation of users' emotion on the Kansei-based web prototype is critical in validating the usefulness of the web design standards that has been proposed in study [1].

\section{LITERATURE REVIEW}

\subsection{Emotion in Web Design}

The use of emotion in human-computer interaction is highly important as it affects human cognition and perception [14]. The involvement of emotions in computer systems design, termed as "affective computing", enhances the computers' ability to 'make decisions' [15], thus associating emotional intelligence in machines. This includes the machine's ability to perceive and respond to emotion, convey emotion, as well as handle the emotion of others [14]. Designing a functional website that is appealing and emotion evoking is typically done visually. The design of the websites establishes first impression and influences users' trust and decision-making. Users tend to reject and mistrust websites with insufficient emotional elements. Websites should be functional, have pleasing aesthetics, and provide emotional engagement to optimize users' experience, and ultimately leading to user satisfaction. Researchers have suggested that affect and emotion in information dissemination is more important than functional outcomes such as usefulness and ease of use [16].

Research focusing on the utilitarian and hedonic dimension of evaluation suggested that users are not only concerned about functionality and usability, but also during the human-computer interactions [17] [18]. Better human-computer interaction can be achieved through incorporating emotional components in web design. Emotion can strongly affect user experience as it triggers user's attention and subconscious evaluation about a product website, environment or interface [17]. User satisfaction could also be enhanced through designs that minimize negative emotions such as anger, frustration, annoyance, as well as confusion. Systematic website with professional look and feels allows for ease of navigation thus increasing website functionality [19]. These induce sense of security, credibility, perceived ease of use, and trust. Conversely, a poorly designed website will stimulate feelings of disgust, increasing the likelihood of users hitting the back button or closing the browser just to avoid unpleasant interaction with the website [18]. This causes user dissatisfaction that will lead to avoidance behavior. Scholars [3] have emphasized the importance of incorporating emotional aspects in web design. Designing based on users' requirements has been a common practice in web design, with much consideration being placed on increasing website's functionality. This leaves limited attention on users' emotional requirements; a significant element of an ideal website design [3].

A product should satisfy various needs, namely functionality, usability, and pleasure [18]. A product should be able to function well, and its usability is achieved if users find the product easy to use. A product can bring pleasure to the consumer when it brings joy and happiness to them [3]. Similarly, an easy-tonavigate website can be said to bring pleasure to the users with attention-grabbing designs that evoke positive emotions [20]. Websites that are able to arouse emotion such as excitement has been proven to be more successful than those who do not. Products (including websites) that are laden with emotional cues persuades consumers to buy, own, and use them [3]. Therefore, it is essential for all three levels (functionality, usability, pleasure) to be embedded in website designs. Emotional elements also have the ability to influence users' preference in selecting websites. These designs offer better user experience that will encourage repeat usage [2]. Moreover, aesthetically-pleasing objects tend to capture users' attention even when they are lacking in functionality. Users are more likely to choose a website that is emotionally appealing over a similar website that provides equal usability and functionality.

\subsection{Geneva Emotion Wheel}

The Geneva Emotion Wheel (Figure 1) pioneered by Scherer [21] has served as a tool for selfreported emotional assessment. Past study [22] indicated that the central constitution of emotion is intrinsically subjective and can only be appraised by self-response tools such as the GEW. The two major approaches; discrete and dimensional were merged to form the self-report measure to assess emotions. GEW incorporates discrete emotions that are aligned in a circular structure in two dimensional spans [21]. The two 
dimensions; valence and control meticulously align discrete emotions in circles according to their emotion families. The valence dimension comprises of negative and positive emotions while the control dimension denotes low and high power. The two dimensions (valence and control) diverges in a circularly arranged emotions that is separated into four quadrants: positive/low power, positive/high power, negative/low power and negative/high power [22]. The control dimension can also be designated as a power dimension (control/power) [22]. The emotion terms in GEW are labelled with ordinary layman languages to ease the understanding of the emotions by the respondents. GEW incorporates response options for each emotion category with different shape of circles indicating different intensity levels. The high intensities were symbolized with bigger circles and are aligned towards the edge of the wheel. While, the low intensity response options were represented with smaller size circles and are arranged towards the center of the wheel. The GEW provides the respondents with two alternative response options which are "no emotion" and "other emotions" as a free response style. The measurement tool is designed with user friendly graphical user interface (GUI) in order to assist the respondent to easily utilize the instrument for self-response evaluation.

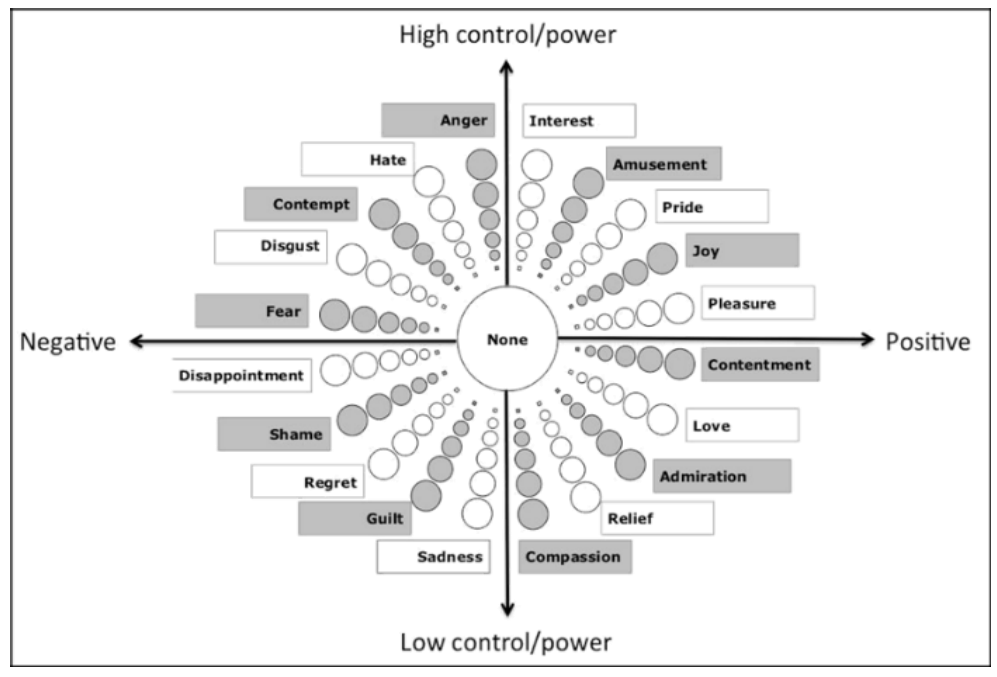

Figure 1. GEW version 3.0

GEW comprises of free response style, discrete emotion reporting, and a multi-dimensional concept of emotions [22]. The "other emotion" option in GEW indicates the free response style allowing respondents to convey other emotions freely during assessment. Studies indicate that pure free response method can be troublesome in emotion measurement process with the presence of diverse responses from the respondents as they freely convey their emotions in their own terms, thus reducing the certainty of the emotion evaluation, making it difficult to be analyzed [22]. Such problems of the free-response style are enhanced by GEW, as the "other emotion" option allows one response along with the existing emotion terms in the wheel. The discrete emotions that are aligned in a systematic circular form refer the discrete response approach in GEW. GEW allows the respondents to express the emotions they feel corresponding to the emotions in the wheel in accordance with the different level of intensities (high to low). The use of ordinary layman language for the emotion terms eases the respondents to understand the emotions in GEW. Moreover, the responses are easier to be interpreted and analyzed [22]. The arrangement of emotions in accordance with the dimensions is visible in GEW compared to the other discrete emotion measurement instruments such as the Differential Emotion Scale [23] and Product Emotion Measuring Instrument (PrEmo) [24].

The presence of the valence and control dimensions signifies the dimensional approach in GEW. The dimensional approach may seem easier for respondents as it requires them to respond to the available dimensions instead of answering in various discrete terms. Furthermore, discrete terms may be challenging for respondents as the dimensional approach is considerably abstract. Although the results are easy to be interpreted and analyzed, the dimensional approach provides lowest delicate results compared to other approaches. The blend of discrete and dimensional approaches in GEW is simply strategic as discrete emotions are presented in layman language and intensifies measurement particularity, whereas, the presence of the dimensional approach assists to detect the emotions in the tool which in turn magnifies usability [22].

GEW has been used in various fields to study users' emotion. [25] employed GEW to study the users' emotions towards the Internet videos. The respondents were asked to convey the emotions they felt 
before and after watching the Internet videos by labelling the videos and selecting three out of the 36 emotional descriptors from GEW [21] in accordance with different intensity levels. [25] stated that the use of GEW was easier for the participants to express their emotional states and their preferences in videos. [26] studied emotions of body movement with the application of GEW. A comparative study was done and the emotional interpretation from an animated character and real actor was explored. The research utilized GEW with twenty emotion labels. The participants were required to report their emotions towards the two clips shown, one with the real actor and another with the animated character. The study reported that the emotions towards the clip of the animated character were perceived as less natural.

Additionally, [27] and [28] have utilized GEW to study emotions in virtual environments. On the other hand, [29] employed GEW to study the affective meaning of listener vocalizations. [30] have studied emotions in interaction with superiors in workplace and [31] explored the emotions of individual in team level and during decision making [31] assured that the GEW can be used under time pressured emotional measurement and for repetitive evaluations. [32] compared the utility of the GEW with the PrEmo [24]. The study attempted to discover the strength and the weakness of the both emotion measurement instrument and to determine the suitability of the instruments in their research field. The study selected two products, designers coffee machine for positive emotions and an alarm clock for negative emotions and conducted research with selected participants. The research explored whether the participants could express their emotions in accordance with the two instruments and also investigated the participants' memory while expressing the emotions. The study revealed that the participants were able to express their emotions with GEW better than the PrEmo as the GEW comprises of more range of emotions and the emotions are presented with the different intensity levels in systematic circular structure. [32] found out that PrEmo has weaknesses such as lack of presentation of the animation in a clear form, the three point scale in PrEmo was considered insufficient for the participants to express their emotions and PrEmo comprises of limited number of emotions which restricts the participants from conveying their feelings thoroughly. The study discovered that the participants favour GEW rather than PrEmo and indicated GEW as an easy tool to use, understand and distinguish between emotions and claimed that the GUI of GEW is attractive [22].

\section{RESEARCH METHOD}

A standard Kansei-based web design guideline for Malaysian higher learning institutions that was developed in [1] was used in a design prototype of a higher learning institution website. Universiti Malaysia Sabah (UMS) website was chosen as a case study and the website was embedded with findings from [1]. The designed Kansei-based website (https://punithathiru92.wixsite.com/mysite) was tested to justify the identified standard Kansei-based web design guideline in [1]. Users' emotional experience towards the Kansei-based UMS website was evaluated through this research with the intention to validate the guideline proposed in [1] to be utilized in design practices. The developed Kansei-based prototype was evaluated through an online GEW survey.

\subsection{Online Survey Design}

An online GEW survey was developed to evaluate users' emotional experience. The online survey was designed in two sections; Section A: Emotion Evaluation with GEW and Section B: Demographic. Section A was designed to gauge the users' emotional experience towards the Kansei-based UMS website. Section B was designed to obtain demographic information of the respondents. The link of the UMS website prototype was included in the online survey to allow respondents to explore the website prior answering the online survey. The online GEW survey used a five-point scale ranging from 1 "low intensity" to 5 "high intensity" .The emotion terms were arranged in accordance to the order in GEW. As for the "other emotions" option as in GEW a short answer text field was added for the respondents to express the emotions they experienced which was not in the list of GEW. The "no emotion" option was included with the five point scale which can be chosen by the respondents if they did not feel a particular emotion in the GEW. The illustration of the online GEW survey is presented in online (https://goo.gl/forms/BWYSUHSQuQ58gFxE3).

\subsection{Participants and Procedure}

This study targeted users of higher learning institution websites as the subjects for the evaluation. A total of 143 respondents participated in the survey. The survey was distributed online through email and social media and was available to be answered for the duration of three months. Face to face sessions were also carried out simultaneously in UMS. The participants were requested to view the developed Kansei-based UMS website for a few minutes by clicking the link provided in the survey and then they were requested to answer the survey questions. They also were provided with a list of definitions used in GEW to assist them in understanding the emotion terms. 


\section{RESULTS AND DISCUSSION}

The survey gathered the data of users' emotional experience in accordance with the GEW. The users' emotional experiences measured on 20 emotions from GEW were evaluated, analyzed with descriptive statistics and are discussed briefly in the sections below. Since the GEW was measured on a five-point scale indicating different intensity level of the emotions ( 5 = High Intensity and 1 = Low Intensity), the mean was calculated to find the average of the gathered users' emotional experience data as reported in Figure 2. Figure 2 showed users' emotional experiences after exploring the Kansei-based UMS website. Since, the GEW includes a center segment with the option 'no emotion', the option was implemented with a scale 'no emotion felt' as ' 0 '. So, they have selected the option of 'no emotion felt' when they did not experience the particular emotion in the list. Figure 3 illustrates the participants' emotional experience towards the Kansei-based UMS website. The data were analyzed with descriptive statistics in order to validate the identified standard guideline in [1] and to justify whether it is acceptable in web designing practices. The mean was used as in the study [6] to calculate the average data and were analyzed based on the average.

\begin{tabular}{|l|c|c|c|c|c|c|}
\hline Emotions & $\begin{array}{c}\text { Intensity } \\
5\end{array}$ & $\begin{array}{c}\text { Intensity } \\
4\end{array}$ & $\begin{array}{c}\text { Intensity } \\
3\end{array}$ & $\begin{array}{c}\text { Intensity } \\
2\end{array}$ & $\begin{array}{c}\text { Intensity } \\
1\end{array}$ & $\begin{array}{c}\text { No } \\
\text { Emotion } \\
\text { Felt }\end{array}$ \\
\hline Interest & 39 & 58 & 36 & 3 & 4 & 3 \\
\hline Amusement & 16 & 35 & 50 & 6 & 4 & 32 \\
\hline Pride & 35 & 55 & 37 & 3 & 5 & 8 \\
\hline Joy & 32 & 47 & 45 & 8 & 4 & 7 \\
\hline Pleasure & 34 & 51 & 41 & 5 & 6 & 6 \\
\hline Contentment & 29 & 52 & 38 & 6 & 6 & 12 \\
\hline Love & 29 & 46 & 34 & 15 & 6 & 13 \\
\hline Admiration & 42 & 40 & 36 & 14 & 5 & 6 \\
\hline Relief & 21 & 44 & 44 & 12 & 3 & 19 \\
\hline Compassion & 16 & 44 & 43 & 8 & 5 & 27 \\
\hline Sadness & 8 & 14 & 19 & 31 & 15 & 56 \\
\hline Guilt & 6 & 16 & 18 & 28 & 16 & 59 \\
\hline Regret & 6 & 18 & 14 & 25 & 20 & 60 \\
\hline Shame & 5 & 15 & 16 & 26 & 20 & 61 \\
\hline Disappointment & 7 & 14 & 20 & 24 & 21 & 57 \\
\hline Fear & 4 & 15 & 15 & 24 & 24 & 61 \\
\hline Disgust & 5 & 12 & 15 & 25 & 20 & 66 \\
\hline Contempt & 4 & 15 & 17 & 25 & 21 & 61 \\
\hline Hate & 4 & 13 & 10 & 27 & 22 & 67 \\
\hline Anger & 6 & 10 & 15 & 26 & 21 & 65 \\
\hline
\end{tabular}

Figure 2. Users' emotional experience relation with GEW

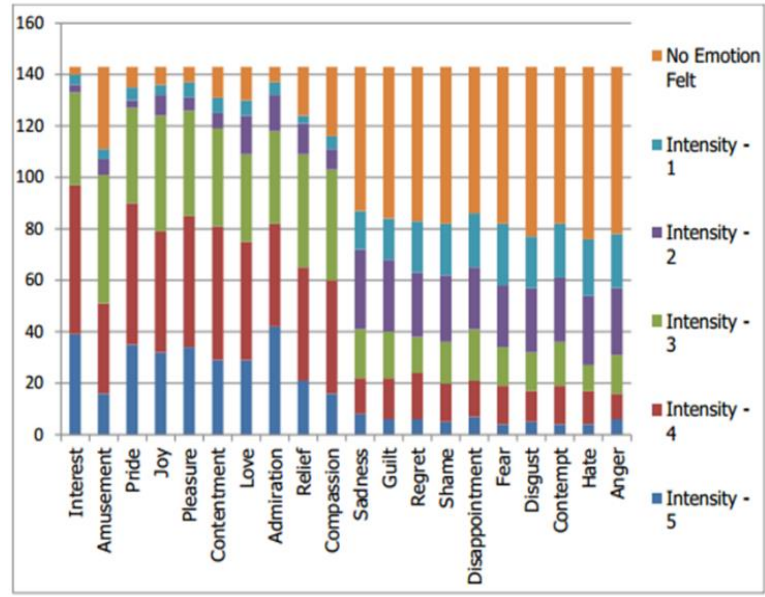

Figure 3. Participants' emotional experience in relation with GEW

The Figure 4 shows the average of each emotion with their different level of intensities. The range for each intensity level was calculated and shown in the Table 1 . The data was analyzed in relation with the range of each intensity levels. Since, this study used five point scale including an extra scale with the score ' 0 ' for no emotion felt option, the range size for each level of intensity was calculated using the formulae [(n1) / n]. Therefore, the range size was [(6-1)/6] which result $5 / 6$ with 0.83333 . The range was identified by adding the range size to the each intensity score.

The data were analyzed in accordance to the mean and the range of each intensity level in order to find the emotions elicited by the Kansei-based UMS website. The emotions experienced by the users while using the website were analyzed and are discussed with the information in Figure 5. Respondents' active participation in the emotional experience evaluation suggests that the Kansei-based UMS website elicits positive emotions from the users. The responses showed that the higher learning institution website prototype evokes positive emotion formation. The report illustrates that the website induced seven out of the ten positive emotions with high intensity. Whereas, the ten negative emotions were evoked at the lowest intensity. Three positive emotions that were stated as neutral in Figure 5 indicates that the emotions were somewhat felt but not with highest intensities. The data showed that the respondents did not feel any negative emotions with high intensity, which means that the majority of the respondents experienced the negative emotions with a very low intensity. 


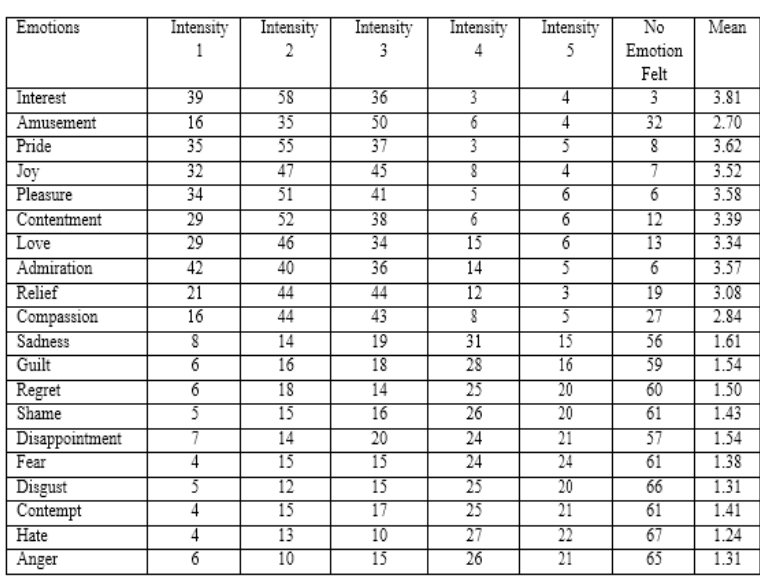

Figure 4. Average of emotions

\begin{tabular}{|l|c|c|c|l|}
\hline Emotions & Mean & Range & Intensity & Emotion State \\
\hline Interest & 3.81 & $3.33-4.15$ & 4 & High Intensity \\
\hline Amusement & 2.70 & $2.50-3.32$ & 3 & Neutral \\
\hline Pride & 3.62 & $3.33-4.15$ & 4 & High Intensity \\
\hline Joy & 3.52 & $3.33-4.15$ & 4 & High Intensity \\
\hline Pleasure & 3.58 & $3.33-4.15$ & 4 & High Intensity \\
\hline Contentment & 3.39 & $3.33-4.15$ & 4 & High Intensity \\
\hline Love & 3.34 & $3.33-4.15$ & 4 & High Intensity \\
\hline Admiration & 3.57 & $3.33-4.15$ & 4 & High Intensity \\
\hline Relief & 3.08 & $2.50-3.32$ & 3 & Neutral \\
\hline Compassion & 2.84 & $2.50-3.32$ & 3 & Neutral \\
\hline Sadness & 1.61 & $0.84-1.66$ & 1 & Lowest Intensity \\
\hline Guilt & 1.54 & $0.84-1.66$ & 1 & Lowest Intensity \\
\hline Regret & 1.50 & $0.84-1.66$ & 1 & Lowest Intensity \\
\hline Shame & 1.43 & $0.84-1.66$ & 1 & Lowest Intensity \\
\hline Disappointment & 1.54 & $0.84-1.66$ & 1 & Lowest Intensity \\
\hline Fear & 1.38 & $0.84-1.66$ & 1 & Lowrest Intensity \\
\hline Disgust & 1.31 & $0.84-1.66$ & 1 & Lowest Intensity \\
\hline Contempt & 1.41 & $0.84-1.66$ & 1 & Lowest Intensity \\
\hline Hate & 1.24 & $0.84-1.66$ & 1 & Lowest Intensity \\
\hline Anger & 1.31 & $0.84-1.66$ & 1 & Lowest Intensity \\
\hline
\end{tabular}

Figure 5. Users' emotional experience in relation with GEW

Table 1. Range of each intensity level

\begin{tabular}{cc}
\hline Intensity Level & Range of Intensity \\
\hline 5 & $4.16-5.00$ \\
4 & $3.33-4.15$ \\
3 & $2.50-3.32$ \\
2 & $1.67-2.49$ \\
1 & $0.84-1.66$ \\
0 & $0.00-0.83$ \\
\hline
\end{tabular}

The analyzed data showed the most positive emotions (highlighted in Figure 5) with high intensities and all negative emotions with the low intensities, it can be inferred that the Kansei-based UMS website elicits positive emotions in users. The result of the users' emotional experience justified the standard Kanseibased web design guideline for higher learning institution websites in [1] and proved that the identified standard guideline evokes positive emotions in users. From the result of the Figure 6, users' emotion model with the seven positive emotions which were experienced with high intensity by the majority of the participants were identified as a result of the users' emotional experience. Table 2 illustrates users' emotion model that was identified through the users' emotional experience evaluation of the Kansei-based UMS website that was designed in accordance with the identified standard Kansei-based guideline with detail design features as in [1]. All seven emotions in the users' emotion model are positive valence, indicating the Kansei-based UMS website cultivates positive emotional engagement with the users.

Table 2. Users' Emotion Model

\begin{tabular}{ccc}
\hline Emotion & Control/Power & Valence \\
\hline Interest & High & Positive \\
Pride & High & Positive \\
Joy & High & Positive \\
Pleasure & High & Positive \\
Contentment & Low & Positive \\
Love & Low & Positive \\
Admiration & Low & Positive \\
\hline
\end{tabular}

The users' emotion model certifies the standard Kansei-based web design guideline in [1] can be successfully used in designing practices of higher learning institution websites. This was proved with the users' emotion model consisting of seven positive emotions with four of high control/power and three of low control/power which indicates positive users' emotional experience towards the Kansei-based UMS website. Users' positive emotional engagement with a website is very important as previous researchers have argued that a website should able to encourage emotional engagement and elicit positive impressions in order to arouse revisit to the website [7]. Users' emotion model from Table 3 is demonstrated in Figure 6. 


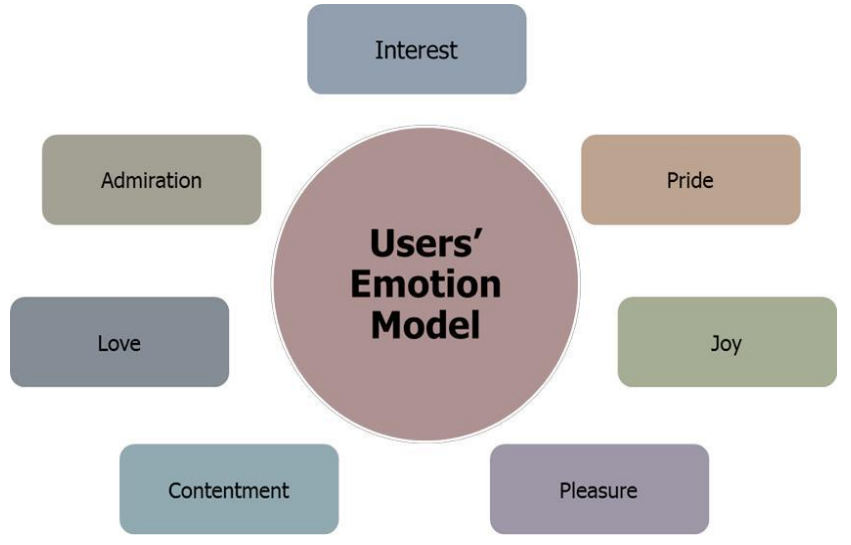

Figure 6. Users' Emotion Model

Seeing the result of positive emotions that elicited towards the Kansei-based UMS website which was developed considering the standard Kansei-based web design guideline for higher learning institutions in Malaysia as in study [1], this research has justified the standard Kansei-based web design guideline can be used in the development of Malaysian higher learning institution websites since it was proved to satisfy the users emotionally with the positive emotional engagement as shown in users' emotion model which presented in Figure 7.

\section{CONCLUSION}

The users' emotional experience evaluation using a self-response method was carried out to justify the identified standard Kansei-based web design guideline for Malaysian higher learning institutions from [1]. The identified guideline with detail design features as in [1] was implemented into current UMS website as a prototype. The prototype was tested to verify that the identified guideline with design details can be used in design practices of higher learning institution websites. The users' emotional experience evaluation has proved that the prototype does cultivate positive emotional engagement between users with the identification of users' emotion model which is presented in Figure 7. The users' emotion model with seven positive emotions which were four of high control/power and three of low control/power certified that the standard Kansei-based web design guideline evokes positive emotional experience in users. Therefore, the identified standard Kansei-based guideline from the study [1] can be used to design an emotional website for higher learning institutions in Malaysia.

\section{REFERENCES}

[1] T. Punitha and B. Aslina, "Identifying a User Interface Web Design Standard for Higher Learning Institutions using Kansei Engineering," Indonesian Journal of Electrical Engineering and Computer Science, vol. 11, pp. 90-97, 2018.

[2] M. Carvalho and L. Oliveira, "Emotional Design in Web Interfaces," Observatorio (OBS*) Journal, pp. 14-34, 2017.

[3] T. J. Howard, et al., "Kansei Engineering and Website Design," Proceedings of the ASME 2012 International Design Engineering Technical Conferences \& Computers and Information in Engineering Conference, pp. 1-11, 2014.

[4] J. S. Lerner, et al., "Emotion and Decision Making: Online Supplement," Annual Review of Psychology, pp. 1-14, 2015.

[5] N. K. Chuan, et al., "Kansei engineering for e-commerce sunglasses selection in Malaysia," Procedia - Social and Behavioral Sciences, Elsevier B.V, vol. 97, pp. 707-714, 2013.

[6] C. R. Ramachandiran and N. Jomhari, "Kansei Engineering: Emotion Exploration in Blogs," International Conference on Biometrics and Kansei Engineering, pp. 7-12, 2013.

[7] D. Cyr, et al., "Perceived interactivity leading to e-loyalty: Development of a model for cognitive-affective user responses," International Journal of Human-Computer Studies, vol. 67, pp. 850-869, 2009.

[8] S. Achiche and S. K. Ahmed, "Genetic fuzzy modeling of user perception of three-dimensional shapes Genetic fuzzy modeling of user perception of three-dimensional shapes," Artificial Intelligence for Engineering Design, Analysis and Manufacturing, pp. 93-107, 2011.

[9] F. Noori, et al., "Designing a University web site by Considering Users' Emotion and Using Kansei Engineering," International Conference of Cognitive Science, pp. 66-70, 2015. 
[10] S. C. J. Lim and A. F. Zakaria, "A Collaborative Tagging System Design Framework for Supporting Affective Design," Proceedings of the 2015 IEEE IEEM, pp. 401-405, 2015.

[11] F. Guo, et al., "Optimization Design of a Webpage Based on Kansei Engineering," Human Factors and Ergonomics in Manufacturing \& Service Industries, vol. 26, pp. 110-126, 2016.

[12] A. Hadiana and A. M. Lokman, "Kansei Evaluation in Open Source E-Learning System,” vol. 3, pp. 135-139, 2016.

[13] S. N. Hussin and A. M. Lokman, "Kansei Website Interface Design : Practicality and Accuracy of Kansei Web Design Guideline," International Conference on User Science and Engineering, pp. 30-35, 2011.

[14] I. Lopatovska and I. Arapakis, "Theories, methods and current research on emotions in library and information science, information retrieval and human-computer interaction," Information Processing \& Management, vol. 47, pp. 575-592, 2011.

[15] H. Sun and P. Zhang, "The role of moderating factors in user technology acceptance," International Journal of Human-Computer Studies, vol. 64, pp. 53-78, 2006.

[16] I. Cristescu, "Emotions in human-computer interaction: The role of nonverbal behavior in interactive systems," Revista Informatica Economică $n r$, vol. 2, pp. 110-116, 2008.

[17] F. Spillers, "Emotion as a Cognitive Artifact and the Design Implications for Products that are perceived as Pleasurable," 2004. Available: http://www.experiencedynamics.com/pdfs/published_works/SpillersEmotionDesign-Proceedings.pdf.

[18] G. Huisman and M. Van Hout, "The Development of a Graphical Emotion Measurement Instrument Using Caricatured Expressions: the LEMtool," in C. Peter, et al., "Emotions in HCI-Designing for People," Proceedings of the International Workshop. Fraunhofer, 2008.

[19] K. Karvonen, "The beauty of simplicity," Proceedings of the Conference on Universal usability, New York, NY: ACM, pp. 85-90, 2000.

[20] V. T. Gorp and E. Adams, "Design for Emotion," 2012.

[21] K. R. Scherer, "Emotion, the psychological structure of," in N. J. Smelser and P. B. Baltes, "International Encyclopedia of the Social and Behavioral Sciences," Oxford, Pergamon, 2001.

[22] V. Sacharin, et al., "Geneva Emotion Wheel Rating Study," Geneva, Switzerland: University of Geneva, Swiss Center for Affective Sciences, 2015.

[23] V. Shuman, et al., "The GRID meets the Wheel : Assessing emotional feeling via self-report," Oxford, Oxford University Press, 2015.

[24] P. M. A. Desmet, et al., "Emotional Design: Application of a Research-Based Design Approach,” Knowledge, Technology and Policy, vol. 20, pp. 141-155, 2007.

[25] S. Bardzell, et al., "Understanding Affective Interaction : Emotion, Engagement, and Internet Videos," Proceeding of IEEE International Conference on Affective Computing and Intelligent Interaction, 2009.

[26] A. Beck, et al., "Comparing Perception of Affective Body Movements displayed by Actors and Animated Characters," Symposium on mental states, emotions and their embodiment: Proceedings of the Symposium at the AISB Convention. Edinburgh, UK, 2009.

[27] M. T. Longhi, et al., "An experiment to understand how the affective aspects can be detected in virtual learning environments," CINTED-UFRGS, vol. 7, 2009.

[28] J. M. R. Santos, "Gaia: Intelligient control of virtual environments," Master thesis, 2008.

[29] S. Pammi and M. Schroder, "Annotating meaning of listener vocalizations for speech synthesis," Procedding of 2009 IEEE International Conference on Affective Computing and Intelligient Interaction, 2009.

[30] F. Tschan, et al., "Discrete emotions in interactions with superiors: Some are more role-related, some are more relationship related," in T. Rigotti, et al., "Gesund mit und ohne Arbeit," Lengerich, Pabst Science Publishers, pp. 289-304, 2010.

[31] V. Tran, et al., "Emotions and decision-making processes in management teams: a collective level analysis," Revista de Psichologia del Trabajo y de las Organizaciones, vol. 28, pp. 15-24, 2012.

[32] D. G. Caicedo and M. Van Beuzekom, "how do you feel?" An assessment of existing tools for the measurement of emotions and their application in consumer products research, 2006. 